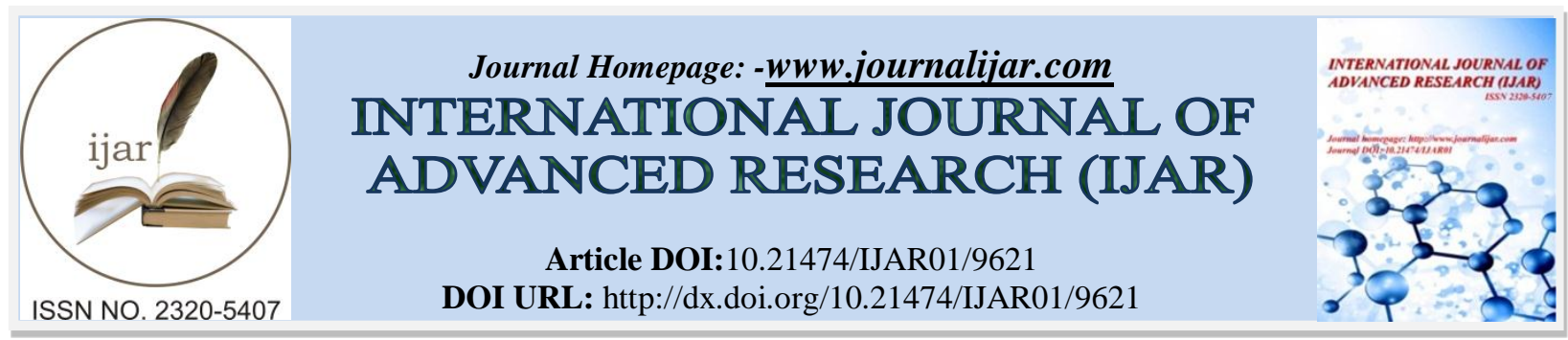

RESEARCH ARTICLE

\title{
ROLE OF LIP PRINTS IN PERSONAL IDENTIFICATION AND CRIMINALIZATION.
}

\author{
Amina Mehrin Bano. \\ Undergraduate student,Saveetha dental college and hospitals,Saveetha institute of medical and technical sciences.
}

\section{Manuscript Info}

Manuscript History

Received: 20 June 2019

Final Accepted: 22 July 2019

Published: August 2019

\begin{abstract}
Establishing a person's identity is a very important process in civil and criminal cases. In the field of forensic sciences, the lip prints have been proven to be an imperative tool in identifying a person positively. Today, in India, as well as worldwide, crimes of diverse character are on the rise. Both criminals and knowledgeable elite of the public are making use of stylish methodological measures while committing crimes to put forensic scientist, police, and the public off the scene. Hence, the role of crime scene detectives has become more challenging than ever in this civilized modern world. Dental, fingerprint, and DNA comparisons are probably the most common techniques, allowing fast and secure identification processes. However, since they cannot always be used, sometimes it is necessary to apply different and less-known techniques. Nevertheless, in certain circumstances related to the scene of the crime or due to the lack of experienced personnel, these techniques might be unavailable; therefore, there is still an increasing need for reliable alternative methods of establishing identity.
\end{abstract}

Copy Right, IJAR, 2019,. All rights reserved.

\section{Introduction:-}

Establishing a person's individuality can be a very difficult procedure. Oral-dental aids, fingerprint, and DNA comparisons are considered as the most common techniques used in this perspective, and it usually permits fast and secure identification processes. Though, they cannot always be used because of expense, availability, and other reasons, sometimes it is necessary to apply different and less known techniques. Investigators often gain information and evidence through the use of odontology, anthropometry, fingerprints, and other techniques that help determine, for example, gender, approximate age, and height. Today, however, investigators may also rely on lip prints to identify possible suspects or to support evidence gained in specific investigations.[1] The wrinkles and grooves on the labial mucosa (called sulci labiorum) form a characteristic pattern called "lip prints," the study of which is referred to as cheiloscopy.[2]Fischer was the first to describe it in 1902. Use of lip prints in personal identification and criminal investigation was first recommended in France by Locard. In 1950, Synder also suggested the idea of using lip prints for identification. Lip prints are considered unique to an individual and analogous to fingerprints. It has been verified that lip prints recover after undergoing alterations such as minor trauma, inflammation, and herpes. However, major trauma to the lips may lead to scarring and pathosis, and surgical treatment rendered to correct the pathosis may affect the size and shape, thereby, altering the pattern and morphology of the grooves. [3] A lip print found at the scene of a crime can be the basis for conclusions with regards to the character of the event, the number of people involved, sexes, cosmetics used, habits, occupational traits, and the pathological changes of the lips

Corresponding Author:-Amina Mehrin Bano.

Address:-Undergraduate student,Saveetha dental college and hospitals,Saveetha institute of medical and technical sciences. 
themselves. Lip prints, as one of the dermatoglyphics, have been used as genetic markers in many congenital and clinical diseases.

\section{Historical implications:}

The biological phenomenon of systems of furrows on the red part of human lips was first noted by anthropologists; R. Fischer was the first to describe it in 1902. Use of lip prints in personal identification and criminalization was first recommended in France by Edmond Locard. In 1950, Synder was the first person who suggested the idea of using lip print for identification. He had conducted an investigation of traffic accident and proved that the characteristics of lips formed by lip grooves are as individually distinctive as the ridge characteristics of fingerprints. Until 1950, however, anthropology merely mentioned the existence of the furrows without suggesting a practical use for the phenomenon. Since 1950, the Japanese have carried out extensive research in the matter. In the period 1968-1971, two Japanese scientists, Y. Tsuchihashi and T. Suzuki, examined 1364 persons at the Department of Forensic Odontology at Tokyo University. Based on this research, it was established that the arrangement of lines on the red part of human lips is individual and unique for each human being.[4] This statement led to the conclusion that there is a possibility of using the arrangement of furrows (on a trace, in a linear form) on lips for the identification of a person. In further research, the Japanese scientists examined the principles of the heredity of furrows on the red part of lips. In Poland, the interest in lip prints started in 1966 when a lip print was revealed on window glass at the scene of a burglary. Research was carried out, and its results were comparable to those achieved in Japan and Hungary. [5] The research was only of preliminary character and, as yet, have not allowed practical application of results. A project aiming at that objective was launched in 1982 in the Forensic Institute of Warsaw University Criminal Law Department in cooperation with the former Forensic Institute of Militia in Warsaw. The material for study was collected in the former Military Training Center at Minsk Mazowiecki. Lip prints were collected from 1500 persons (including 107 women) from various locations around the country. The age of the volunteers varied from 5 to 60 years. Altogether, more than 7000 traces of the red part of the lips were examined. As a result of the examination, the individuality of lines in the red part of lips and their exchangeability within the limits practicable for identification was proven. [6] Since 1985, in Poland, the methods of finding and recovery of lip traces, recovering comparative material, and the techniques employed to carry out that expertise have been introduced into casework of the Fingerprint Department of the Central Forensic Laboratory of Police in Warsaw. During the years 1985-1997, cheiloscopic techniques have been used in 85 cases, including 65 burglary cases, 15 cases of homicide, and five cases of assault. In 34 cases, the identification was positive, indicating that cheiloscopic techniques were equal in value to other types of forensic evidence. It has also been included in evidence for presentation in court.

\section{Cheiloscopy}

Cheiloscopy, the study of lip prints, is a forensic investigation technique that deals with the identification of humans based on lips traces. Lip prints have to be obtained within 24 hours of the time of death to prevent erroneous data that would result from post mortem alterations of lip. Lip print pattern depends on whether mouth is opened or closed. In closed-mouth position, lip exhibits well-defined grooves, whereas, in open position, the groves are relatively ill defined and difficult to interpret.

The basics of cheiloscopy, however, are similar to that of dactyloscopy, i.e. lip prints are consistent, permanent, and permit establishing a classification. [7] The significance of cheiloscopy is frequently linked to the fact that lip prints are distinctive to one person, even in monozygotic twins. Like fingerprints and palatal rugae, lip grooves are permanent and remain steady throughout life. The lips are covered with wrinkles and grooves that forms a characteristic pattern-the lip print. Searching for lip prints in a crime scene investigation can be very important in establishing the true nature of the facts. Lip prints can link a subject to a specific location if found on clothes or other objects, such as glasses, cups, or even cigarette butts. However, visible lip prints are important and those that are not visible are also important. The invisible lip prints are called latent lip prints. Various materials used for developing artificial lip prints forensic investigations related to medico-legal issues are aluminum powder, silver metallic powder, plumb carbonate powder, fat black aniline dyer, lysochrome dyes, and fluorescent dyes.

The vermillion border of the lips has minor salivary and sebaceous glands, which, together with the moisturizing done by the tongue, leads to the possibility of existence of latent lip prints. So, when searching for lip prints, one must always consider the presence of latent lip prints. Domiaty et al. studied in depth the lip prints of Saudi individuals with the aim to explore the relative importance of the lip prints in investigations related to the medicolegal issues in Saudi population. Red or brown lip sticks, white copy papers, and tissue papers were utilized to make 
the impressions of the lips. Every lip print was divided into six topographic areas, examined by magnifying hand lenses, then photographed and wired to the computer to be examined by the picture manager and fax viewer programs. [8] They also recommended establishing a database for all individuals in a certain locality, hoping to be a reference in civil lawsuits and criminal cases.

As regards the predominant pattern among both sexes that the prevalent pattern in males was pattern I while it was pattern III in females with no statistically significant difference between patterns in both sexes. [9] Non significance could have occurred due to small sample size, so larger samples might be needed to clarify degree of significance. This coincides with a study in India by Badiye and Kapoor, but differs from the study by Krishnan et al. which was focusing on the role of lip prints, finger prints for gender differentiation and showed there was a relationship between lip pattern and sex but the predominant pattern in their study was pattern IV in males and I in females. This agrees with Prabhu et al. who showed in their study that there is no correlation between lip pattern and gender as pattern $\mathrm{V}$ was predominant in both sexes. Also a study in Dakahlia (Egypt) showed that the prevalent pattern in both sexes was pattern I negating the correlation between lip pattern and sex. [10]

The research studies and data regarding lip print pattern use in identification are so scanty. The current study was established in order to provide more details regarding the usefulness of lip print pattern, showing its distribution among a sample of Egyptian population. To our knowledge the present study is the first in Egypt to describe lip print pattern among different geographical areas. It was noticed from the current study that no similar pattern was shown between any two individuals and this was in coincidence with Suzuki ET al.and Kumar et al., which supports the role of lip print in personal identification being unique to each person. This review attempts to shows that the most frequent pattern among persons enrolled in it was pattern IV and this agrees with a study done by Prabhu et al. which was done on 100 individuals with the aim of extensive examination of lip print patterns with referral to the frequency of each pattern among them. Sivapathasundharam et al. reached the same result in their study, confirming that the most prominent pattern was IV. [11] Contrasting a study by Kapoor and Badiye showed that the predominant pattern in Indian sample population of 200 persons was pattern I, this could be attributed to the fact of different location and races of involved populations that might be related to genetic factors. Prabhu et al. explained that this variation could be due to geographical variation or strict standards of different pattern It was also observed that no two persons had similar lip prints, either the same type or different types. It was further noticed that not even a single person had one particular type of lip print in the upper lip or lower or in both. [12] Thus the statement of Tsuchihashi is true and can be justified in stating that each of the 750 subjects has his own or her own lip print. Analysis of lip print between twins and their parents and between the twins themselves were studied, the observation is that neither the twins, nor the twins with their parents have similarity of lip print. This observation of the present study is in contradiction to the study made by Tsuchihashi In her study, twins frequently showed patterns extremely similar to those of their parents. [13] At the same time the present study agrees with the study of MacDonell who described two identical twins who seemed to be indistinguishable by every other means but lip prints in these twins differed.

As to the permanence of the lip print the present study agrees with that of the study carried out by Tsuchihashi and his statement "in a criminal search, where the unchanged pattern even for a 6 months period would be helpful" is justified. Ball had reported the history of lip prints and importance of its evidence in the courts and the status of lip prints as a source of Forensic evidence. She had also stated that latent lip prints would be available at all crime scenes as the vermilion borders of lips have minor salivary glands and sebaceous glands with the latter being principally present around the edges of the lip associated with hair follicles, sweat glands in between and secreting oils. It is these secretions and continual moisturizing by the tongue due to occasional sebaceous glands present on the lip to alveolar mucosa, crossing the transitional zone, there are chances for the presence of the latent lip prints on items such as glasses. Through the research carried out by Petersen it was evident that lip prints at crime scenes are rarely mentioned simply due to the fact most investigators or crime scene examiners do not look for them. On the numerous occasions when a smear or a smudge is discovered, most crime scene personnel disregard it as being a fingerprint that is unidentifiable. It is important to note though, lip prints left at scenes of a crime are more prevalent than one thinks. Articles such as drinking glasses, letters, cigarette butts, clothing, napkins and even skin may possess lip prints that could eventually lead to the identity of a suspect, victim or a witness of a crime. In the present study the recording of lip prints in non-biological materials namely in drinking glasses were carried out and the comparison of photographs of the lip prints taken from the glasses and the lip prints collected using the tracing method are found to be the same for the same persons under study. It reveals that the methodology of tracing lip print in glass and stainless steel tumblers using finger print powder is justified. 
It is not easy to position the lip prints in the general system of traces. The unique properties of the lip print help in identifying a human being spatially when it is revealed as a stratified surface trace with visible elements of lines. In the case where the lines are not clear, individual identification of a human being based on this trace is extremely difficult unless the trace contains more individual characteristics (e.g., scars), and the identification often ends with group identification. In these cases, it is possible to examine the substance that constitutes the trace (e.g. saliva) as a biological trace and to determine the blood group in the ABO system. Also, the use of lipsticks is not indispensable for leaving lip prints. The edges of the lips have sebaceous glands, with sweat glands in between. Thus, secretions of oil and moisture from these enable development of "latent" lip prints, analogous to latent fingerprints. Even though the lines and furrows are present both in the upper and lower lip, from one corner of the mouth to the other corner, only the middle portion of the lip is taken into account, since this portion is always visible in any traces.

\section{Conclusion:-}

Lip print analysis is a process that can provides both qualitative and quantitative results; however, more researches should be done to make its application widely accepted in the forensic field. Cheiloscopy deals with the examination of system of furrows on the red part of human lips and is an important tool in forensic science. The investigation of lip prints (both visible and latent) can thus be fundamental in resolving a criminal act. Traditional lipstick produces a print that is easily identifiable. However, lip prints made without lipstick are invisible, requiring reagents that are more sensitive than conventional materials to locate and develop the prints. Research suggests the conclusive evidence that lip prints are suitable for the successful comparison, analysis, and identification of a person to a crime. In fact, there have been convictions of perpetrators who were positively identified via the analysis of their known lip prints to those found at the crime scene. There is a need to develop one cohesive cheiloscopy system that is practicable in forensic odontology.

\section{Reference:-}

1. Synder LM. Textbook of Homicide investigation. Identification of dead bodies. Springfield : Charles C Thomas; 1950; 65.

2. Kasprazak J. Possibilities of cheiloscopy. Forensic Sci Int. 1990; 46: 145-151.

3. Aggrawal A. The importance of lip prints .Web Mystery Magazine. 2004; 11(2): http://lifeloom.com//II2Aggrawal.htm. Visited 5-01-2009.

4. Suzuki K and Tsuchihashi Y. Personal identification by means of lip print. J Forensic Med. 1970; 17(2): $52-57$.

5. Vahanwahal SP and Parekh DK. Study of lip prints as an aid to forensic methodology. J Ind Dent Assoc. 2000; 71: 269-271.

6. Hirth L, Gottsche H, Goedde HW. Lip prints - variability and genetics. Human Genetik. 1975 Oct 20;30(1):4762

7. Tsuchihashi Y. Studies on personal identification by means of lip print. Forensic Sci Int. 1974; 3: $233-248$.

8. Williams TR. Lip prints - Another means of identification. J Forensic Indent. 1991; 41(3): 190-194.

9. Kasprazak J. Cheiloscopy. Encylopedia of Forensic Science. UK : Elseiver; 2000; 358-361.

10. Suzuki K. and Tsuchihashi Y. Two criminal cases of lip print. ACTA Criminol. Japan. 1975; 41: 61-64.

11. Ball J. The current status of lip prints and their use for identification. J Forensic Odontostomatol. 2002; 20(2): 43-46.

12. Synder LM. Textbook of Homicide investigation. Identification of dead bodies. 1950;65.

13. Maheswari TU, Gnanasundaram N. Role of lip prints in personal identification and criminalization. Anil Aggrawal's Internet Journal of Forensic Medicine and Toxicology. 2011 Jan 1;12(1).

14. Caldas IM, Magalhaes T, Afonso A. Establishing identity using cheiloscopy and palatoscopy. Forensic science international. 2007 Jan 5;165(1):1-9.

15. Van Ooschot, R. A. H., and Jones, M. K. (1997) DNA fingerprints from fingerprints. Nature (London) 387,767

16. Alvarez-Segu'1, M., Miquel, M., Castello', A., and Verdu', F. (2000) Persistent lipsticks and their lip prints: new hidden evidence at the crime scene. Forensic Sci. Int. 112, 41-47

17. Castello', A., Alvarez, M., Miquel, M., and Verdu', F. (2002) Long-lasting lipsticks and latent prints. Forensic Sci. Commun.

18. http://www.fbi.gov/programs/lab/fsc/past/verdu.htm 4. Castello', A., Alvarez, M., and Verdu', F. (2002) A new chemical aid for criminal investigation: dyes and latent prints. J. Soc. Dyers Colourists 6, 316-318

19. Walsh, P. S., Metzger, D. A., and Higuchi, R. (1991) Chelex 100 as a medium for simple extraction of DNA for PCR based typing from forensic material. Biotechniques 10, 506-513 
20. Lorente, M., Entrala, C., Lorente, J. A., Alvarez, J. C., Villanueva, E., and Budowle, B. (1998) Dandruff as a potential source of DNA in forensic casework. J. Forensic Sci. 43, 901-902

21. Lorente, J. A., Lorente, M., Wilson, M. R., Budowle, B., and Villanueva, E. (1994) Analysis of short tandem repeat (STR) HUMVWA in the Spanish population. Forensic Sci. Int. 65, 169- 175. 